\title{
A DEFORMABLE SURFACE MODEL FOR SOFT VOLUME SIMULATION
}

\author{
Sylvester Arnab \\ Vinesh Raja \\ Warwick Manufacturing Group \\ University of Warwick \\ CV4 7AL, Coventry, UK \\ E-mail: s.arnab@warwick.ac.uk
}

\section{KEYWORDS}

Surface model, Physically Based Modelling, Mass Spring System, Deformable Model

\begin{abstract}
This paper introduces a surface alternative to the volume model commonly employed in soft object simulation. This is inspired by a significant interest in employing the surface data due to its simplicity. However, there are issues in properties estimation and volume preservation. Therefore, the aim of the ongoing research includes exploring the potential of a surface mass spring model with shape-preserving springs for volume simulation. The approach illustrates the possibility of deriving the spring stiffness at runtime based on the real material properties as well as achieving a global shape deformation despite the non-existence of internal volume. The proposed scheme can be further explored to address other material properties.
\end{abstract}

\section{INTRODUCTION}

There has been an increase in the use of soft volume simulation in medical training, where accurate visual and behaviour replications of human organs are of great importance. These organs are geometrically modelled into 3 dimensional volumes based on medical data such as CT/MRI scans. This volume data can then be simulated based on the organ's physical and material properties. To allow tactile interaction with the visual representation, haptic rendering has been introduced. However, there is a significant interest in utilising surface data as an alternative to volume data due to its simplicity. A surface model has the benefit of having a less complex mesh network compared to the volume counterpart, which can be manipulated to emulate soft volume behaviour. The main issue is that volume simulation requires constant volume preservation as well as correct volume behaviour during simulation. Generally, a surface model would collapse under gravity and without the internal volume, determining the correct deformation effect would be a challenge. Figure 1 shows that the haptic interaction on the cylinder surface does not produce the correct global deformation effect on the opposite side. For instance, a blood vessel with internal pressure from the blood density would produce a deformation effect at the opposite surface relative to the interaction point. The proposed approach explores these issues where, a surface mass spring system with anisotropic shape preserving springs and weighted constraints have been employed.

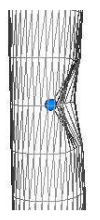

Figure 1: A Surface Model Deformed upon Interaction

\section{RELATED WORKS}

To create a volume model using surface data, the surface model can be re-meshed to create internal volume. However, this creates computational overhead during simulation imposed by a more complex volume network. The addition of new artificial springs (Bourguignon and Cani 2000) to the existing volume mesh produces an object that is stiffer than it should be (Hong et al. 2006). There are also attempts that employ surface data which addressed shape preservation but not volume (Nedel and Thalmann 1998; Aubel and Thalman 2000). (Hong et al. 2006) introduced weighted constraints that control the deformation distribution of the muscle instead of using additional springs. However, this method is fundamentally focusing on the local radius of influence of the interaction and is not influenced by the orientation of the interaction force.

A more effective shape preserving method has been embedded into the Mass Spring Systems (MSS) where the springs are placed at the mesh nodes. They are also known as the local shape memory (Marchal et al. 2005) and zero-length springs. These springs have been employed to simulate non-linear skin behaviour of a virtual thigh (Mendoza et al. 2002; Laugier et al. 2003). (Choi et al. 2005) administered these springs to preserve object shape during simulation when a force is imposed on the nodes. However, the stiffness of the springs was either statistically fine-tuned based on predefined properties (Zhang et al. 2002) or regularly distributed. (Arnab and Raja 2007) extended this 
method by extracting the properties of the springs based on radial links (Vassilev \& Spanglang 2002; Balaniuk and Salisbury 2003).

Volume behaviour is influenced by the properties estimated for the model. Regular properties distribution is very common where the regular mesh topology (regular node concentrations) is assumed (Gelder 1998; Delingette 1998; Bourguignon and Cani 2000; Brown et al. 2001) or in the case of irregular node concentrations, the topology is modified to be as regular as possible (Deussen et al. 1995; Bielser 2003). However, when a portion of the surface model is refined, the regular topology becomes irregular. Consequently, the properties require re-estimation within the refined area. (Choi et al. 2005) attempted properties re-estimation after surface refinement based on the topology but the behaviour patterns between the coarse and the refined area do not coincide. The behaviour is improved by our previous method discussed in (Arnab and Raja 2007) where these patterns achieve a higher level of co-incidence.

\section{THE PROPOSED SCHEME}

The scope of dynamic behaviour in this research is within a constrained space such as a human breast fixed on a static body (figure 2). The model is constructed from the surface mesh and the dynamic behaviour is achieved by employing the surface MSS with volumetric supports.

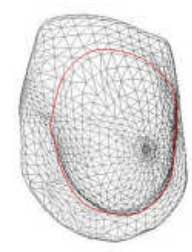

Figure 2: A Breast Model (within the drawn boundary) with Convex Shape

To address the issue of properties estimation as well as the non-existence of inner volume for the proposed surface model, the object's internal volume represented by the surface elements has to be considered. Initial discretisation of volume is required to estimate the properties based on the real material properties. The assumption is that the initial shape of object is convex with known centre of object. This scheme extended the approach described in (Arnab and Raja 2007).

\section{Surface Mesh Topology}

The surface mass spring model is based on the surface mesh topology where the springs are represented by the edges of the triangular elements (figure 3). For instance, the edge that connects the nodes with mass $\mathrm{m}_{\mathrm{i}}$ and $\mathrm{m}_{\mathrm{j}}$ is the spring with stiffness $\mathrm{k}_{\mathrm{ij}}$. For a spring link $\boldsymbol{i} \boldsymbol{j}$ as in figure 2, the internal force $\boldsymbol{F}_{\boldsymbol{i j}}$ is

$$
F_{i j}=K_{i j}\left(\left\|p_{j}-p_{i}\right\|-l_{i j}\right) \frac{p_{j}-p_{i}}{\left\|p_{j}-p_{i}\right\|}
$$

, where $\boldsymbol{i}$ and $\boldsymbol{j}$ denote the indexes for the 2 nodes, $\| \boldsymbol{p}_{\boldsymbol{j}}$ $\boldsymbol{p}_{i} \|$ is the magnitude of the displacement of the current state of the spring link $\boldsymbol{i j}, \boldsymbol{l}_{\boldsymbol{i} \boldsymbol{j}}$ is the rest length of the spring link, and $\boldsymbol{K}_{i j}$ is the stiffness (spring) coefficient of the node pair. The system of equations is solved through the time using numerical integration techniques.

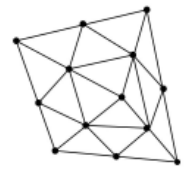

(a)

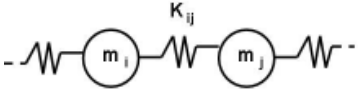

(b)
Figure 3: Surface Mesh Topology: a) the nodes or vertices connected by edges (b) a pair of nodes or masses connected by a spring

This spring network will not be able to preserve volume behaviour during deformation due to the nonexistence of the inner volume mesh. Therefore, inner supports, which are based on the concept of shape preserving springs, are introduced to provide the volumetric reinforcement.

\section{Volumetric Support}

In order to preserve the rest shape of the object, the inner volume support (figure 4) has to be defined for the mass at the nodes. The volume spring works in a similar way as the surface spring but the rest length of the spring is zero. The springs preserve the object shape at equilibrium as they act as virtual anchors.

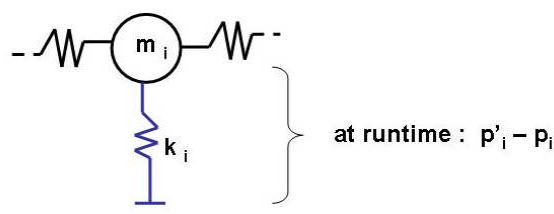

Figure 4: An inner spring stretched during simulation

Based on Equation (1) but rest length is equal to zero, the reaction force at inner spring will only affect mass at node $\boldsymbol{i}$,

$$
F_{i}=K_{i}\left(\left\|p_{i}^{\prime}-p_{i}\right\|\right) \frac{p_{i}^{\prime}-p_{i}}{\left\|p_{i}^{\prime}-p_{i}\right\|}
$$

,where $\boldsymbol{K}_{\boldsymbol{i}}$ is the stiffness of the inner spring at node $\boldsymbol{i}$, $\boldsymbol{p}_{\boldsymbol{i}}{ }_{i}$ is the new position of node $\boldsymbol{i}$ at runtime and $\boldsymbol{p}_{\boldsymbol{i}}$ is the anchored position of node $\boldsymbol{i}$ (figure 4). In order to not only preserve the object shape but also maintain a constant object volume during simulation, the concept of these shape preserving springs has been extended. 


\section{Volumetric Discretisation}

The relationship of the surface elements to the global object properties is defined and employed to extract the material properties for each of the surface elements of a mass spring mesh. Explicit inner volume approximation is carried out for the underlying surface mesh. Initially, the relationship of the surface nodes relative to the centre of object (Vassilev \& Spanglang 2002; Balaniuk \& Salisbury 2003; Arnab \& Raja 2007 ) is considered (figure 5).

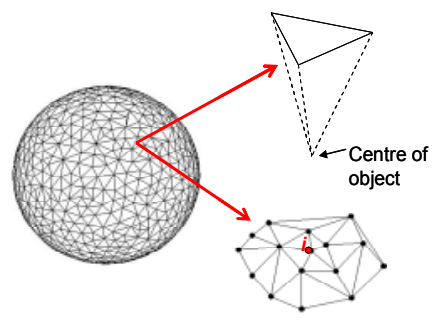

Figure 5: Volumetric Discretisation based on Radial Links

If the mesh is of irregular topology, the triangles are of different sizes. Therefore, different properties should be distributed to the individual triangles. The obvious issue here is the need to correctly distribute the properties from the neighbouring triangles to the member nodes. For instance, mass at node $\boldsymbol{i}$ is determined by manipulating the masses at the neighbouring triangles of which it is a member. (Bourguinon and Cani 2000) and (Bielser 2003) uniformly distributed mass to the respective nodes based on the barycentric relationship. In the case of a triangle, a third of the triangle mass is distributed to the nodes. Each node accumulates the mass contributions from the neighbouring volume represented by the triangle.

However, since the mass at the nodes are not known, the barycentric position of the triangle is determined by assuming uniform mass at the nodes. This is of course incorrect if the nodes are of different individual distances to the object centre. Hence, the proposed volume discretisation is based on the stress and strain relationship of a material instead. This relationship has been employed to extract the elasticity modulus of a real material and is based on a force parallel to the normal of the cross-sectional area. In MSS, the internal and external forces will directly influence the dynamic behaviour of the nodes and not the triangular elements. Therefore, as illustrated in figure 6 , the new distance vector with length $\boldsymbol{L}^{\prime}{ }_{i}$, relative to the object centre, the initial length $\boldsymbol{L}_{\boldsymbol{i}}$ and the surface normal at the node, can be derived.

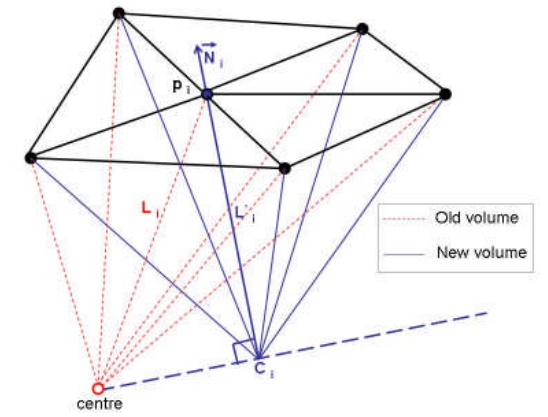

Figure 6: Volumetric Discretisation based on the Neighbouring Triangles Relative to the Normal at $\boldsymbol{p}_{\boldsymbol{i}}$

The new volume is based on node $\boldsymbol{p}_{\boldsymbol{i}}$ relative to the new centre point $c_{i}$ and the other nodes or vertices of the triangular element. Hence, the properties of a node are directly influenced by its normal and the volume relative to its normal. This discretisation supports the need for the volume springs at the nodes to be influenced by the orientation of force relative to the normal. Similar discretisation and volume calculation will be carried out for the other nodes relative to their respective normals and neighbouring triangles. The resulting discretisation represents the mass and the material properties within the volume.

\section{Mass Estimation}

To support both irregular topology and objects with non-uniform shape, the mass distribution is based on the volume discretisation in figure 5 . The total mass of the virtual model has to replicate the total mass of the real object. Therefore, the estimation method has to take mass preservation into account. This has to be true even when the mesh topology is modified. Therefore, the mass at node $\boldsymbol{i}$ is estimated as

$$
m_{i}=\frac{\sum_{i \in t} v_{t}}{\sum_{i=0}^{n} v_{i}} M
$$

, where the estimation ratio for each node is the total volume under the neighbouring triangular elements $t$ (of which node $i$ is a member) divided by the total volume for all $\boldsymbol{n}$ nodes, and $\boldsymbol{M}$ is the object mass. This method can be used to re-estimate mass upon any topological modifications.

\section{The Estimation of Spring Stiffness}

The surface spring stiffness is treated like a membrane which behaviour mimics the surface of an object. Therefore, the spring stiffness is distributed based on an estimation algorithm which has been commonly employed to estimate the behaviour of a membrane with irregular mesh topology (Gelder 1998; Maciel et. al 2003; Lloyd et al. 2007; Arnab and Raja 2007)

The volume spring stiffness defines the level of elasticity of virtual space or volume represented by the 
node, which is also based on the explicit volume discretisation. Therefore, the value of the stiffness is influenced by the normal at the node. The elasticity of the stiffness can be described based on the elasticity, shear and bulk modulus. These characteristics are considered when the estimation method is derived in order to address the issue of the single dimensionality of the existing spring and to extend its role from not only to preserve the object shape at equilibrium but also to dynamically maintain the object volume during simulation.

The behaviour of the spring stiffness at runtime depends on the orientation of the acting force along each spring. Based on this relationship and the fact that the properties at the node $\mathrm{i}$ have been extracted based on its normal, figure 7 illustrates the orientation of the force $\mathrm{F}_{\mathrm{i}}$ relative to its normal $\mathrm{N}_{\mathrm{i}}$. The stiffness of the volume spring $\boldsymbol{K}_{\boldsymbol{i}}$ at each node $\boldsymbol{i}$ is derived at runtime:

$$
K_{i}=\left[\left\|\vec{N}_{i} \cdot \vec{F}_{i}\right\| 1-\left\|\vec{N}_{i} \cdot \vec{F}_{i}\right\|\right]\left[\begin{array}{l}
K_{E} \\
K_{G}
\end{array}\right]_{i}
$$

, where $\left\|\vec{N}_{i} \cdot \vec{F}_{i}\right\|$ is the dot product of the normal unit vector and the acting force unit vector at node $\boldsymbol{i}, \boldsymbol{K}_{\boldsymbol{E}}$ and $\boldsymbol{K}_{\boldsymbol{G}}$ are the spring stiffness based on linear elasticity modulus and shear (rigidity) modulus respectively.

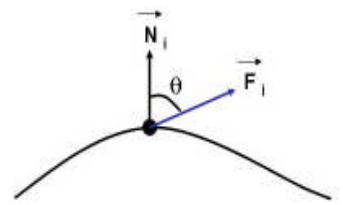

Figure 7: Force Vector relative to the Normal Vector

The stiffness dimension based on the 2 extremities is initialised before the simulation. The dimension is defined as

$$
\left[\begin{array}{l}
K_{E} \\
K_{G}
\end{array}\right]_{i}=E \sum_{i \in t} \frac{V_{t}}{L_{i}^{\prime 2}}\left[\begin{array}{c}
1 \\
1 / 2(1-v)
\end{array}\right]
$$

, where $\boldsymbol{E}$ is the Young's Modulus and $\boldsymbol{v}$ is the Poisson Ratio of the material. The anisotropic extraction illustrates the possibility of employing multidimensional inner spring stiffness.

\section{Volume Behaviour}

In order to preserve volume during simulation, the spring dimension can be extended to address other properties such as bulk elasticity as a factor against volume variation during simulation. Bulk stiffness $\boldsymbol{K}_{\boldsymbol{B}}$ at node $i$ is:

$$
K_{B i}=\frac{E}{3(1-2 v)} \sum_{i \in t} \frac{V_{t}}{L_{i}^{{ }^{2}}}
$$

Volume displacement during simulation can be derived based on the volume calculation employed in
(Hong et al. 2006). This calculation will be correct even when the surface becomes concave during simulation. Therefore, force at node $\boldsymbol{i}$ along its normal unit vector at a time step without any external force interaction on the surface is

$$
F_{i}+=K_{B i} \Delta V w_{i} \vec{N}_{i}
$$

where, $\boldsymbol{\Delta} \boldsymbol{V}$ is the volume displacement, and $\boldsymbol{w}_{\boldsymbol{i}}$ is the weighted constraints that control the distribution of the volume penalty force. The constraint is generally set to 1 , which means the volume change affects all nodes equally but constrained by their respective bulk stiffness. This arrangement produces a global deformation as shown in figure 1 .

Therefore, in order to correctly distribute the interaction force effect to the object surface, the weighted constraints have to be correctly distributed based on the interaction radius of influence where, the sum of the constraints is equal to the number of nodes. The radius of influence (Hong et al. 2006) has been modified and the interaction force orientation is introduced as the correction factor. If the surface nodes are within the radius of influence $r$, weight at node $i$ is

$$
w_{i}=\left(\frac{p_{i}-p_{f}}{\left\|p_{i}-p_{f}\right\|} \cdot \vec{F}_{f}\right)\left(\cos \left(\frac{\left.\left\|p_{i}-p_{f}\right\|^{*} * \Pi\right)}{r 2}\right)\right)
$$

where, $\boldsymbol{p}_{\boldsymbol{i}}$ and $\boldsymbol{p}_{\boldsymbol{f}}$ are the position vector of node $\boldsymbol{i}$ and the position of where force is imposed respectively. Since the sum of weighted constraints is equal to the number of nodes (Hong et al. 2006), the constraint values have to be normalised.

Upon interaction, the direction of the volume penalty force at node $\boldsymbol{i}$ depends on the position of node $\boldsymbol{i}$ relative to the point of interaction. Therefore, based on Equation (3) and (4), the penalty force at node $i$ is

$$
F_{i}+=K_{B} \Delta V w_{i} \frac{p_{i}-p_{f}}{\left\|p_{i}-p_{f}\right\|}
$$

\section{EXPERIMENTAL FINDINGS}

The framework for the empirical experiments has been implemented on top of Microsoft Visual C++, OpenGL and OpenHaptics platforms. Phantom Desktop haptic device has been employed to provide the interaction and the desktop PC has the specification of Intel Pentium 4, $2.40 \mathrm{GHz}$ and $1 \mathrm{G}$ RAM. To evaluate the estimation method, four different distribution schemes have been compared:

- Scheme A : Uniform mass and inner stiffness

- Scheme B : Irregular mass and regular inner stiffness

- Scheme C : Irregular mass and inner stiffness (single stiffness dimension) 


\section{- Scheme D : The Proposed Scheme}

Further comparisons are carried out against the Finite Element Model (FEM) and volume MSS (VMSS). The elasticity modulus is re-extracted from the model to evaluate if the proposed model emulates the same material behaviour.

\section{Properties Estimation}

The stiffness of the springs is based on the real elasticity properties of the object material. Therefore, to evaluate the proposed properties estimation scheme (Scheme D), the Young's modulus (E) can be derived from the strain and stress relationship of the proposed deformable model and compared to the original $\mathrm{E}$. The same experiment is repeated for other surface mesh complexities. The values (Table 1) demonstrate that the deformable surface model can closely emulate the volume material behaviour.

Table 1: The Young's Modulus (E) extracted from the model when a constant force is imposed on the surface of a cube

\begin{tabular}{|l|l|l|l|}
\hline $\begin{array}{l}\text { Model } \\
\text { (complexity) }\end{array}$ & $\begin{array}{l}\text { Cube } \\
(602 \\
\text { nodes })\end{array}$ & $\begin{array}{l}\text { Cube } \\
(1352 \\
\text { nodes })\end{array}$ & $\begin{array}{l}\text { Cube } \\
(2402 \\
\text { nodes })\end{array}$ \\
\hline E (100 Pascal) & 100.07 & 98.7 & 98.7 \\
\hline E(3.25 Kpa) & 3.30 & 3.17 & 3.11 \\
\hline
\end{tabular}

To evaluate the re-estimation of properties, a local surface area is refined and the properties are then reestimated. A constant force is imposed on a node and the displacement data is collected. The displacement patterns within the coarse and the refined area are compared (Choi et al. 2005; Arnab and Raja 2007). If the patterns are identical, the deformation behaviour is preserved despite the change in the mesh topology. The displacement behaviours are studied where 2 values are analysed:

i) The standard deviation between the 2 patterns determines their level of co-incidence. The smaller the standard deviation, the more identical the patterns are

ii) The mean deviation of the 2 patterns represents the error in behaviour after refinement. The least value indicates the least deviation from the original behaviour

Figure 8 describes the findings based on a sphere model with irregular mesh topology to illustrate the concept.

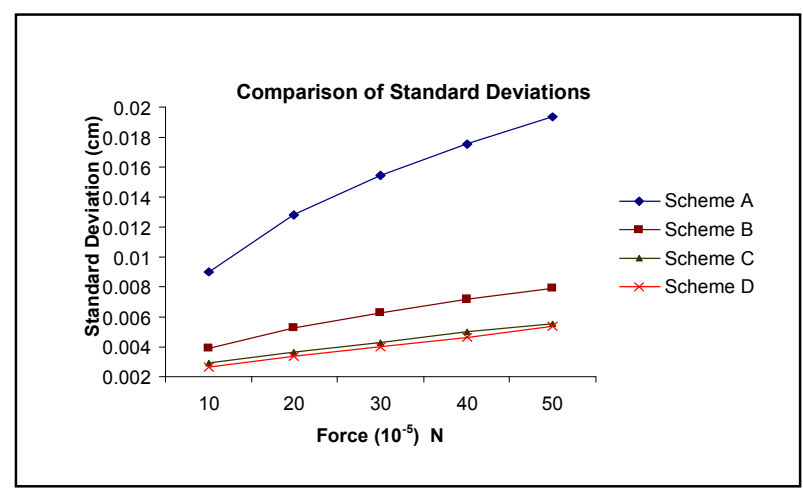

(a)

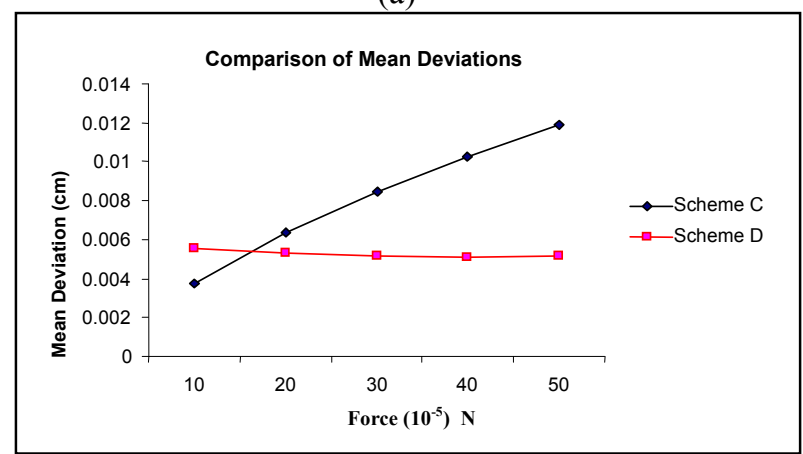

(b)

Figure 8: Properties Re-estimation Test ( $\mathrm{E}=10$ Pascal):

(a) The standard deviation of the patterns $(\mathrm{C}$ and $\mathrm{D}$ denotes the most co-incidence) (b) Average displacement deviation comparison, where $\mathrm{D}$ maintains the least error

The standard deviations comparison in 8(a) shows that both scheme $\mathrm{C}$ and $\mathrm{D}$ produce high level of coincidence between the displacement patterns. However, scheme D maintains a minimal displacement deviation for any magnitude of force compared to scheme $\mathrm{C}$ where its deviation increases with force $(8$ (b)). Hence, the analysis concludes that the proposed scheme D preserves the properties and the local dynamic behaviour with minimal standard deviation when a local area is refined. Tests have been carried out on models with various mesh complexities and they also draw similar conclusions

\section{Shape and Volume Preservation}

To evaluate volume preservation during simulation, the global behaviour is observed. Figure 9 shows that the complete irregular scheme improves the surface behaviour with gravity.

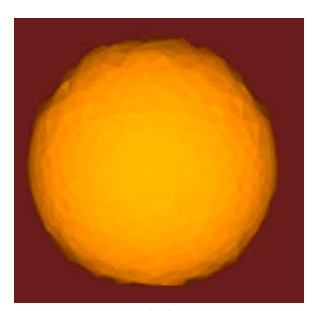

(a)

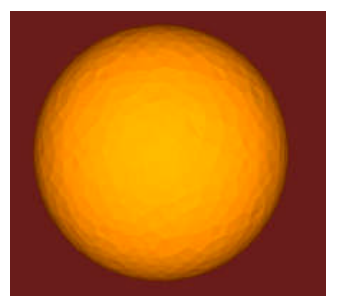

(b)
Figure 9: Gravity test: (a) Scheme B (b) Scheme D 
When a constant force is imposed on the surface, the object shape changes and deforms. The analysis concludes that the irregular scheme preserves the object volume with the least volume deviation as illustrated by the average deviation percentage comparison in figure 10.

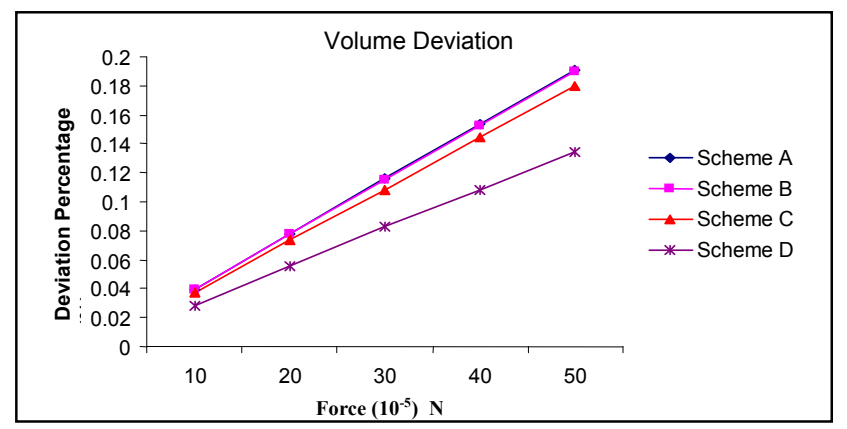

Figure 10: The percentage of the average volume deviation

\section{Global Deformation Effect}

To produce a more realistic global deformation based on the interaction force radius and orientation of influence, the weighted constraints are manipulated at runtime. When these constraints are set to 1 (figure 11 (a)), the global deformation as in figure 11 (a) is produced. Upon interaction, the weighted constraint at each node is changed during simulation in regards to the radius and the interaction force orientation at runtime. Consequently, figure 11 (b) shows that at runtime, the automatic constraint derivation based on the force orientation and radius of influence produces a more realistic behaviour.

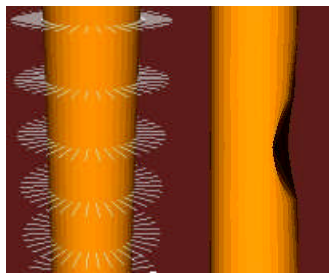

(a)

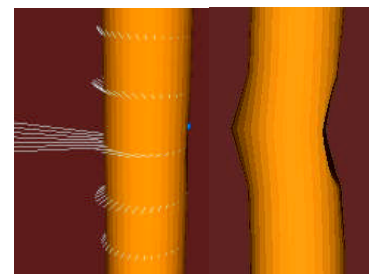

(b)
Figure 11: The white lines reflect the weighted constraints at the nodes (a) constraints are uniform (b) constraints extracted at runtime

Models such as cubes with different surface mesh complexities have been compared with the FEM model as analysed by SolidWorks/Cosmos. A shear force is imposed on the top surface while the opposite surface is fixed. When compared with FEM, the shape produced is similar with minor deviation (figure 12). The displacements of the selected nodes from the same location on both models also indicate minimal and subtle deviation.

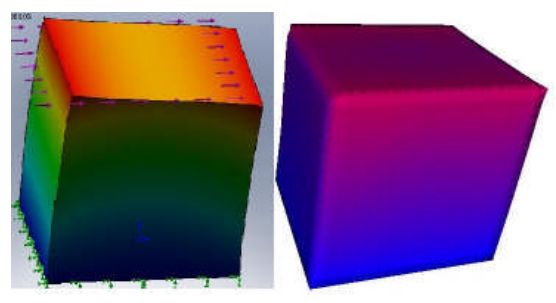

Figure 12: Shape comparison (a) FEM (b) Proposed Scheme

\section{Performance}

A simple performance test has been carried out to illustrate that the surface data reduces both mesh and computational complexity. Table 2 shows that the average frame per second (FPS) achieved by the VMSS and the proposed surface model.

Table 2: Total FPS Comparison

\begin{tabular}{|c|c|c|c|c|}
\hline $\begin{array}{c}\text { Model } \\
\text { (number of } \\
\text { surface } \\
\text { nodes) }\end{array}$ & $\begin{array}{c}\text { Cube } \\
(602 \\
\text { nodes })\end{array}$ & $\begin{array}{c}\text { Cube } \\
(1352 \\
\text { nodes })\end{array}$ & $\begin{array}{c}\text { Cube } \\
(2402 \\
\text { nodes) }\end{array}$ & $\begin{array}{c}\text { Sphere } \\
(1000 \\
\text { nodes })\end{array}$ \\
\hline VMSS & 77 & 37 & 20 & 47 \\
\hline Scheme D & 80 & 44 & 25 & 77 \\
\hline
\end{tabular}

\section{CONCLUSIONS}

This paper has illustrated the feasibility of employing a surface model to simulate soft solid. The proposed scheme introduces volumetric discretisation based on the node normal, the anisotropic extraction of inner spring relative to force orientation as well as weighted constraints to control global deformation effect. Local deformation behaviour is preserved regardless of the mesh resolutions and global deformation effect is achieved despite of the non-existence of internal volume. It displays similar behaviour with the FEM model and produces minimal deviation from the original elasticity modulus. Furthermore, the computational performance is better than the volume counterpart. Further evaluations such as the perception test will be carried out to verify the visual and haptic feedback experienced by the users. The scheme is currently explored in the ongoing research in breast simulation (figure 13).
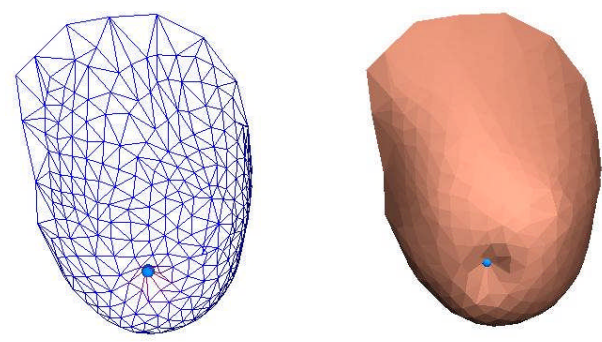

Figure 13: A Breast Model Deformed Upon Interaction 


\section{REFERENCES}

Arnab S.; Raja V. 2007, Irregular Surface Mesh Topology for

Volumetric Deformable Model, The $4^{\text {th }}$ INTUITION International Conference and Workshop, 4-5 October 2007, Athens, Greece

Aubel A. and Thalmann D. 2000, Realistic Deformation of Human Body Shapes, Proc. Computer Animation and Simulation 2000, Interlaken, August 2000, pp. 125-135.

Balaniuk R. and Salisbury K. 2003, Soft Tissue Simulation using the Radial Element Method, IS4TM-International Symposium on Surgery Simulation and Soft Tissue Modelling, France, June 12-13 2003

Bourguignon D. and Cani M. P. 2000. Controlling Anisotropy in Mass-Spring Systems, Computer. Animation and Simulation 2000, 113-123

Bielser D. 2003. A Framework for Open Surgery Simulation, Doctor of Technical Sciences Thesis, Swiss Federal Institute of Technology, ETH, Zurich, 2003

Brown J.; Sorkin S.; Bruyns C. 2001. Real Time Simulation of Deformable Objects: Tools and Application, In Comp. Animation, 2001

Choi Y.; Hong M.; Choi M.; Kim M. 2005. Adaptive Surface-deformable Model with Shape-preserving Spring, Journals of Computer Animation and Virtual Worlds, Comp. Anim. Virtual Worlds 2005, number 16, pages $69-83$

Delingette H. 1998. Towards Realistic Soft Tissue Modeling in Medical Simulation, Proceedings of the IEEE: Special Issue on Surgery Simulation, 512-523, April 1998

Deussen O.; Kobbelt.; Tucke P. 1995. Using Simulated Annealing to Obtain Good Nodal Approximations of Deformable Objects, Computer Animation and Simulation '95, Springer-Verlag, 1995

Gelder A. V. 1998. Approximate Simulation of Elastic Membranes by Triangulated Spring Meshes, Journal of Graphics Tools 1998, 3(2), pages 21-41

Hong M.; Jung S.; Choi M.; Welch S. 2006. Fast Volume Preservation for a Mass-Spring System, IEEE Computer Graphics and Applications, September/October 2006, 83-91

Laugier C.; Mendoza C.; Sundaraj K 2003. Towards a Realistic Medical Simulator using Virtual Environments and Haptic Interaction, Robotics Research: The Tenth International Symposium, Springer Tracts in Advanced Robotics Volume 6/2003

Lloyd B. A.; Szekely G.; Harders M. 2007, Identification of Spring parameters for Deformable Object Simulation, IEEE Transactions on Visualisation and Computer Graphics, Vol. 13, No. 5, Sept/Oct 2007

Maciel A.; Boulic R.; Thalmann D. 2003, Towards a Parameterization Method for Virtual Soft Tissues Based on Properties of Biological Tissue, In International Symposium on Surgery Simulation and Soft Tissue Modeling, 2003

Marchal M.; Promayon E.; Troccaz J. 2005. Simulating Complex Organ Interactions: Evaluation of a Soft Tissue Discrete Model, ISVC 2005, LNCS 3804, pages 175182, (C) Springer-Verlag Berlin Heidelberg 2005

Mendoza C.; Sundaraj K.; Laugier C. 2002. Issues in Deformable Virtual Objects Simulation with Force Feedback, International Advanced Robotics Program
(IARP): International Workshop on Human Robot Interfaces, Rome - Italy, 2002.

Nedel L. P. and Thalmann D. 1998. Real-Time Muscle Deformations Using Mass Spring Systems, Proc. Computer Graphics Int'1, IEEE Press, 1998, pages 156165

Vassilev T. and Spanlang B. 2002, A Mass-Spring Model for Real Time Deformable Solids, Proceedings of EastWest Vision 2002, pp. 149-154, Graz, Austria, September 12-13, 2002

Zhang J.; Payandeh S.; Dill J. 2002. Haptic Subdivision: an Approach to Defining Level-of-Detail in Haptic Rendering, Proceedings of the 10 Symposium on Haptic Interfaces for Virtual Environment and Teleoperator Systems, Orlando, FL, March, 2002, pages 201-208

\section{AUTHOR BIOGRAPHIES}

SYLVESTER ARNAB obtained an Honours Degree in Computer Science at The University of Manchester in 1998 and a masters degree in Distributed Multimedia Systems at the University of Leeds in 2001. He has worked at University Malaysia Sarawak (UNIMAS) in Malaysia as a lecturer and researcher for 5 years. He is currently reading a doctorate degree at the University of Warwick. His research interest is in deformable models for medical simulations. His email address is: s.arnab@warwick.ac.uk

VINESH H. RAJA is currently a professorial fellow at the University of Warwick and is currently leading the Informatics Research group within the Warwick Manufacturing Group. His research interests are applications of ICT in healthcare sector. He focuses on augmenting and extending everyday, learning and work activities with interactive technologies that move 'beyond the desktop'. This involves designing enhanced user experiences through appropriating and assembling a diversity of technologies including haptics, handheld and pervasive computing. The main focus of his research is not the technology per se but the design and integration of the digital representations that are presented via them to support social and cognitive activities in ways that extend current capabilities.His e-mail is: raja_v@wmgmail.wmg.warwick.ac.uk. 